Article

\title{
Detection of Potentially Compromised Computer Nodes and Clusters Connected on a Smart Grid, Using Power Consumption Data
}

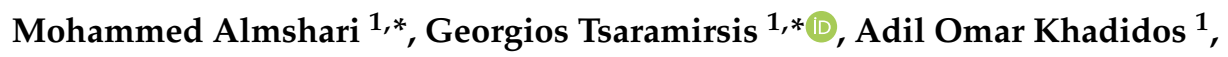 \\ Seyed Mohammed Buhari ${ }^{1}$ (D), Fazal Qudus Khan ${ }^{1}$ (D) and Alaa Omar Khadidos ${ }^{2}$ \\ 1 Department of Information Technology, Faculty of Computing and Information Technology, \\ King Abdulaziz University, Jeddah 21589, Saudi Arabia; akhadidos@kau.edu.sa (A.O.K.); \\ mesbukary@kau.edu.sa (S.M.B.); fqkhan@kau.edu.sa (F.Q.K.) \\ 2 Department of Information Systems, Faculty of Computing and Information Technology, \\ King Abdulaziz University, Jeddah 21589, Saudi Arabia; aokhadidos@kau.edu.sa \\ * Correspondence: malmshari0002@stu.kau.edu.sa (M.A.); gtsaramirsis@kau.edu.sa (G.T.)
}

Received: 18 July 2020; Accepted: 4 September 2020; Published: 7 September 2020

check for updates

\begin{abstract}
Monitoring what application or type of applications running on a computer or a cluster without violating the privacy of the users can be challenging, especially when we may not have operator access to these devices, or specialized software. Smart grids and Internet of things (IoT) devices can provide power consumption data of connected individual devices or groups. This research will attempt to provide insides on what applications are running based on the power consumption of the machines and clusters. It is therefore assumed that there is a correlation between electric power and what software application is running. Additionally, it is believed that it is possible to create power consumption profiles for various software applications and even normal and abnormal behavior (e.g., a virus). In order to achieve this, an experiment was organized for the purpose of collecting $48 \mathrm{~h}$ of continuous real power consumption data from two PCs that were part of a university computer lab. That included collecting data with a one-second sample period, during class as well as idle time from each machine and their cluster. During the second half of the recording period, one of the machines was infected with a custom-made virus, allowing comparison between power consumption data before and after infection. The data were analyzed using different approaches: descriptive analysis, F-Test of two samples of variance, two-way analysis of variance (ANOVA) and autoregressive integrated moving average (ARIMA). The results show that it is possible to detect what type of application is running and if an individual machine or its cluster are infected. Additionally, we can conclude if the lab is used or not, making this research an ideal management tool for administrators.
\end{abstract}

Keywords: smart grid; power consumption; IoT; machine learning; security; privacy; descriptive analysis; F-Test of two samples of variance; two-way analysis of variance (ANOVA); autoregressive integrated moving average (ARIMA); malware detection

\section{Introduction}

Unwanted applications cause many hurdles on easily accessible computers in the lab. Some common hurdles, such as slowness of the computers and security risks are caused due to unwanted background processing. Sometimes the machines maybe used remotely or at certain times (e.g., while a lab is closed) run applications that are not supposed to. It is common for users to use the computational power of company machines for personal benefits at the expense of their organization (e.g., cryptocurrency mining, hosting applications, etc.). In some cases, privacy rules may make 
it difficult to conduct active monitoring of what applications the users are running. Furthermore, users may use their personal machines in order to avoid detection since the administrators will have no access to these machines. However, organizations have full access to their smart power grid and they have every right to monitor the power consumption for the various computer/clusters or even rooms [1]. Smart grids have a number of advantages such as unit/cluster power consumption monitoring, automatic control by including hardware and software monitoring and controlled applications [2]. Additionally, it is possible to monitor the power consumption in real time [3].

This research will attempt to detect if a machine and a cluster of machines is infected by unwanted applications, such as a virus. It will also attempt to predict what applications/types of applications are running on the machine based on power consumption data. Finally, we can detect possible occupancy with is traditionally done by use of multiple sensors and applications [4]. The novelty of this work can be summarized by the following objectives:

(1) Detect if a node and/or a cluster is infected;

(2) Differentiate between different types of applications;

(3) Detect occupancy of a node and/or a cluster.

This is not the first attempt to use power consumption data in order to produce diagnostics. Several researchers have focused on the use of power consumption data and used machine learning algorithms to analyze them. Some of the applied research in this field has focused on various applications in different domains such as smartphones, computers and home electricity.

Luckett et al. [5] proposed a new method for detecting any root-kit behavioral process. They were able to achieve high accuracy and high training speed due to the small size of the data set. Their data was collected from the computer processor power consumption with sample period of five minutes, then various machine learning algorithm were used and tested the method on more than one operating system to ensure that it will have high accuracy. An experiment conducted by Jimenez et al. [6] focused on collecting the power consumption data of the computer processing unit with the network data. They then used ten machine learning techniques to analyze and classify if a computer was infected or not. They used a software to generate high utilization to add more features to avoid wrong alarms. They successfully detected malwares using both network and power data using the Random Forest algorithm. Another experiment proposed by Loanes et al. [7] aimed to evaluate and detect the abnormal state of the power grid by measuring the power losses information and observe the deviation between the normal and abnormal state using neural networks techniques. The proposed monitoring and detecting method used real world data and also simulated data. This method was able to detect the abnormal behavior. Mehrotra et al. [8] proposed a method to classify the applications running in the smartphone using the power consumption data. This method classified the applications into three categories: low, medium and high. Their dataset was collected by installing an application in the smartphone to collect the consumption data. Then all data were divided into three types based on the power consumption. Classification was conducted by using five different techniques. Their method was applied on 77 applications and managed to successfully classify them as low, medium and high based on their power consumption. Kurniawan et al. [9] developed a detection method for Android smartphone operating system. The method was based on generating a dataset with sample period of one minute from the power consumption, the temperature of the battery and network traffic of the mobile. An application was used to log all the power data related to the battery. The researchers then classified the data, using four different machine learning algorithms. Their method achieved an accuracy of $85.6 \%$ with the Support Vector Machine (SVM) algorithm. According to Zeffere et al. [10], no access is needed to identify and detect the malwares. In their work, they proposed a methodology that detects the abnormal activities by analyzing the energy usage of an Android smartphone. Their dataset was generated by using a software which collects the consumption data with a sample period of $250 \mathrm{~ms}$. Two techniques were used to classify the data and detect the malwares. This method detected malwares with $87 \%$ accuracy. Abykoon et al. [11] suggested a method which can 
identify what is the device connected to the power using the data of power consumption and machine learning techniques. Their dataset was collected with a sample period of five minutes. Classification in this research was using different supervised and unsupervised techniques. Each device had a unique signature that can be used to classify what type of device it is. However, in some cases and due to the similarities, more training was required in order to achieve high accuracy.

Prediction of the needed power is important to ensure the stability of the power. Moon et al. [12] proposed a method that predicts the needed power using machine learning techniques. Their data were collected for one year with a 15-min sample period. In their model, they used two machine learning algorithms: Artificial Neural Networks and Support Vector Regression. The results of their approach show that Artificial Neural Networks had better accuracy than the Support Vector Regression. In many cases, the usage of the power differs between home use and industrial use, especially in terms of the rates and prices. Organizations such as electricity companies are trying to identify the behavior of the used power in order to ensure stable network and also differentiate between residential and commercial usage. Shao et al. [13] developed an energy consumption prediction model using Support Vector Machines to provide an estimation of the power consumption. Their dataset was collected by installing four sensors to measure the power, temperature, water flow and humidity. High accuracy prediction of the power consumption was successfully achieved and it included predictions such as possible high-power consumption during holidays at hotels and resorts. A method proposed by Gajowniczek et al. [14] attempted to identify if it is a household usage or not. Their dataset was provided by an electricity company for more than four thousand households with a thirty-minute sample period. They used different machine learning algorithms but they concluded that Support Vector Machine produced the best results with higher accuracy compared to the other algorithms. Markovič et al. [15], from the strategies of designing the electricity network, used classification techniques to predict the amount of power that will be needed in the future in order to avoid rebuilding the infrastructure. They collected a dataset of one year of power usage and they created different user profiles to classify the household users. Iqbal et al. [16] proposed an IoT based architecture capable of exploring electrical devices in smart homes, using sensors, load balancing and data processing. This architecture was tested on six hours of dataset and was proven successful. Data-driven models were used by Bourdeau et al. [17] to predict power consumption with different machine learning techniques and also used different techniques for data preprocessing. This is because it is mainly affecting the accuracy of the prediction results. Input data origin and variables of the dataset also affected the accuracy of the prediction results. The data were analyzed and divided into eight different types: building characteristics and operation information, deep-learning-based time series, mathematical characteristics, past time-steps/data points, time-related indicators, occupancy, indoor environment, and outdoor environment. The researchers concluded that different machine learning techniques were different for each scenario, with each of them having its own advantages and disadvantages.

Croce et al. [18] developed a new technique to monitor power consumption for smart buildings in which user privacy is respected. The architecture was designed as a distributed peer-to-peer to monitor power consumption, and connected with the nearest node, giving multiple peer-to-peer connections. Instead of using individual data, they clustered them to preserve privacy. However, the method had other features such as load control, energy prediction, and voltage stabilization. In this research, ready residential building datasets were used, and the sample period was of one week.

A new method was proposed by Zhang et al. [19] to monitor power consumption and then to store and analyze these data using machine learning. By utilizing the central processing unit (CPU), the consumption changed. Validation was completed by testing and simulating an attack that was intended to make a change on the CPU in order to create and simulate an utilization, and then the method could efficiently detect the change of power consumption with high accuracy and privacy. The authors used different simulation techniques and then analyzed and verified that the method is achieving high accuracy. 
Youngjun and Young [20] proposed a new sensor that can measure high surges. Protection from surges is an advantage of this sensor in which it will represent the duration and lifetime of the protection with the help of an application that is used along with the sensor. The main idea of this research is to ensure that power is stable without any extra surge that affects the electrical device and will be damaged if it exceeds the normal limits. In this research, the sensor was designed as hardware with a small liquid-crystal display (LCD) screen in a small plastic enclosure box. The experiment was implemented using a surge simulator to increase the current for testing the sensor.

Blazakis et al. [21] discussed a new detection method for analyzing the power consumption data and then identify if there was any user that was not authorized and returned to fraud activity. An existing dataset was used in this research, which was generated from a real usage of more than five thousand houses for the duration of almost two years and the sample period for the dataset is half an hour. The results showed that this method has a successful detection rate compared to other methods, and almost in every scenario the method detected the unauthorized usage of the electricity. More than ten scenarios were tested to verify the success of this method.

Analysis and comparison of power consumption in different buildings were proposed by Cibinskiene et al. [22]. The importance of the energy savings and how to reduce energy consumption, especially in workplaces concerning previous researches to ensure continuity of the energy were studied. The regression analysis proved that saving energy will make a difference if the behavior of the staff focused on saving energy goals, and behavior was the most effective part for changing power consumption at residential or workplaces.

Jooseok Oh [23] discussed the use of smart technologies under the Internet of things that can save energy. Once home users can monitor and see the consumption of each device in their home, they will get more knowledge about the consumption of different devices, and this knowledge helps to shut down the devices that consume a lot of power and are not used, such as heating devices and air conditioners. A study was done by Jooseok to give Internet of things devices and training to home users to see if they can reduce usage and achieve power consumption. Home users successfully reduced the power consumption with the help of the Internet of things and the smart plugs, and this confirms that if users can see the real monitoring of the home appliances, they will manage their usage efficiently by using a timer and scheduling the time to start and stop.

Threats of Internet of things (IoT) devices have been highlighted by Myridakis et al. [24]. In this research, the dataset from the IoT devices was used with a detection system along with the difference in power current from the power supply to identify if there was an attack. One of the advantages is that this technique has the lowest costs. The experiment included a device that is simulated to be attacked by changing the hardware as an attacker change. The results of the experiment concluded with successful identification of security attack for the smart IoT devices as it is designed with basic features that may have an attack due to the simplicity.

Moradzadeh et al. [25] proposed a technique to monitor and analyze the power consumption data for the disaggregation and for calculating the expected power needed for the future for specific devices. The dataset was drawn from real data for power consumption captured for home electrical usage. The sample of the dataset was two days. Using the machine learning technique dataset was analyzed and validated the concept of the power consumption data, we can estimate the total needed power for each electrical device.

Forecasting electricity consumption using autoregressive integrated moving average (ARIMA) among industries at Guangdong province in China was performed [26] Three ARIMA models were considered to forecast electricity consumption. The research showed that ARIMA $(1,1,1)$ provided results which are precise and could predict effectively. Similarly, variation in energy consumption for South Africa was predicted using ARIMA, Nonlinear Grey Model (NGM) and NGM-ARIMA models [27].

In order to achieve our objectives, we collected real power consumption data from a computer cluster of two machines as well as the consumption data from each machine, of a university computer 
lab for $48 \mathrm{~h}$, using power monitoring sensors. In parallel, we obtained data from these machines regarding what applications are running during the experiment. During the last $24 \mathrm{~h}$, we infected one of the machines with a custom virus (described in Section 2) in order to observe the variations in the power consumption. Upon completion, the three datasets were merged into one and processed using different machine learning techniques for the purpose of identifying associations between what was happening in the machines/cluster and the power consumption.

Making predictions based on power consumption profiles for applications is challenging due to the nondeterministic nature of the problem. A machine is running multiple applications and each application can consume different amount of power at different times based on activities. Attempting to speculate what is happening in a cluster of multiple computers adds additional complexity to the problem.

The rest of the paper is structured in the following way: Section 2 explains the experiment and data collection process in detail. Section 3 analyzes the data using various approaches. Section 4 concludes the work while providing directions for future research in this area.

\section{Materials and Methods}

This section explains the materials and methods relevant to each of the three proposed research objectives.

\subsection{Experimentation Setup}

In order to answer the research questions presented in the introduction, we generated our own dataset, which included various system characteristics that were collected while using the system with and without viral infection. The dataset associated what application was running and its corresponding power consumption. Both software and hardware solutions were utilized for this purpose.

The experiment utilized a computer cluster consisting of two Dell 9020 Desktop machines with dedicated NVIDIA GeForce 210 GPU cards. The machines were connected to three power consumption measurement sensors. Each machine and the cluster were connected to one SONOFF POW R2 sensors with the ESPurna firmware [28]. All machines and sensors were identical. A Raspberry Pi3 with NodeRed [29] acted as a MQTT broker server that will collect real-time data every second from the SONOFF sensors. The Sensors were connected to the Pi3 via Wi-Fi. Figure 1 illustrates the topology schematic of the experiment used for data collection and Figure 2 shows the connectivity between the devices.

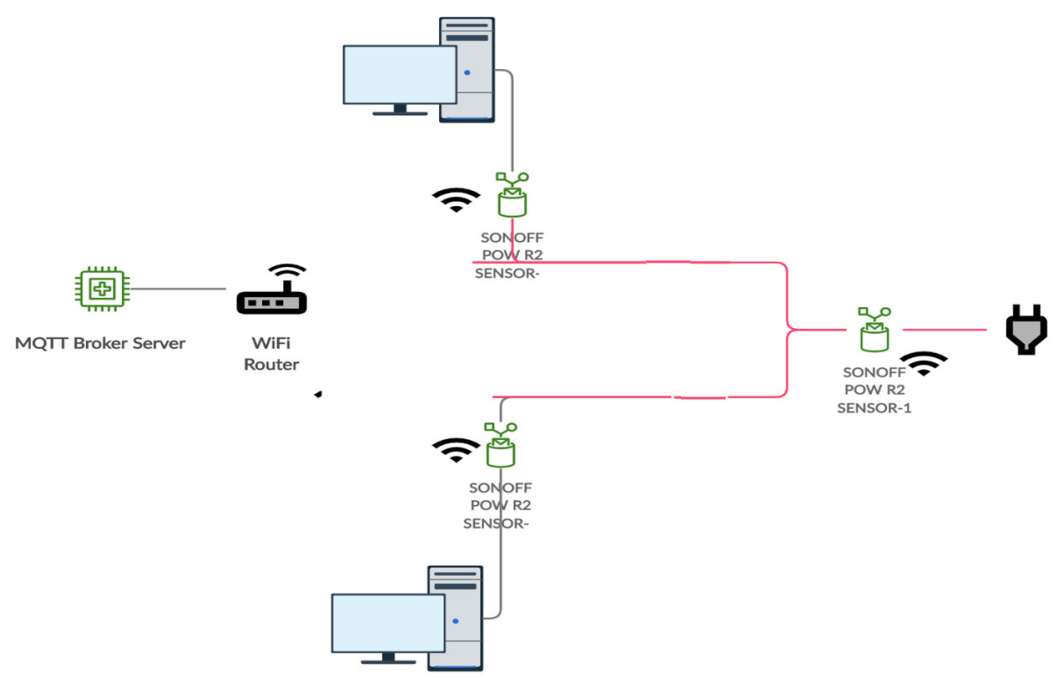

Figure 1. Experiment topology schematic diagram. 

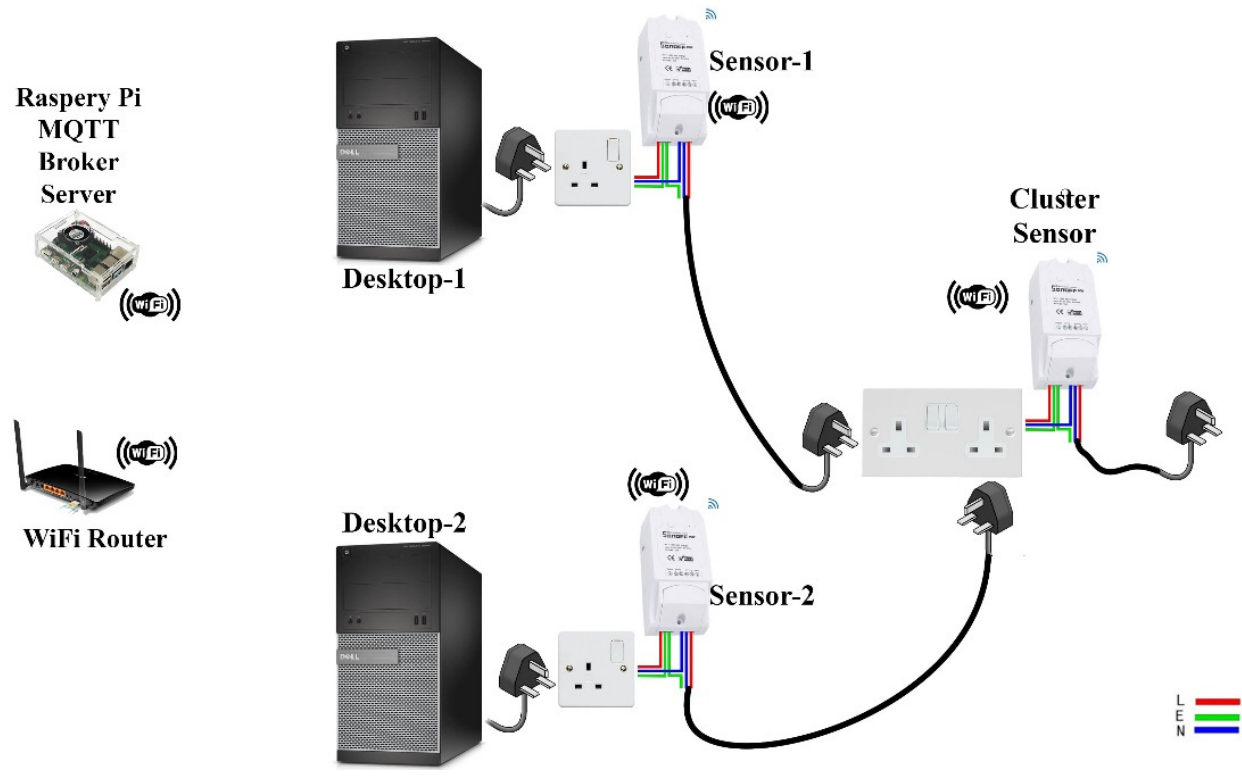

Figure 2. Experiment device connectivity.

The various devices participating in the experiment as well as their connectivity is illustrated in Figure 2. Table 1 presents the schedule of the applications that were executed during the experiment. As it can been seen from Table 1, various applications were run for different times segregated from each other. The different run durations (e.g., an application running for one hour and another one for $30 \mathrm{~min}$ ) increases the difficulty of the analysis but it represents a more realistic usage.

During the second day at 11:30 AM, we infected desktop number 2 with a virus generated by the Virus Maker tool [30] and continued running the applications as normal. The Virus Maker tool has the ability to add multiple behaviors to infected files. However, in this experiment we were mainly interested in behaviors that potentially have impact to the power consumption. Such behaviors include, "random activity", "infinity message boxes", "opening of random files" and "slowing down of the computer". Figure 3 shows the options selected during the generation of the virus.

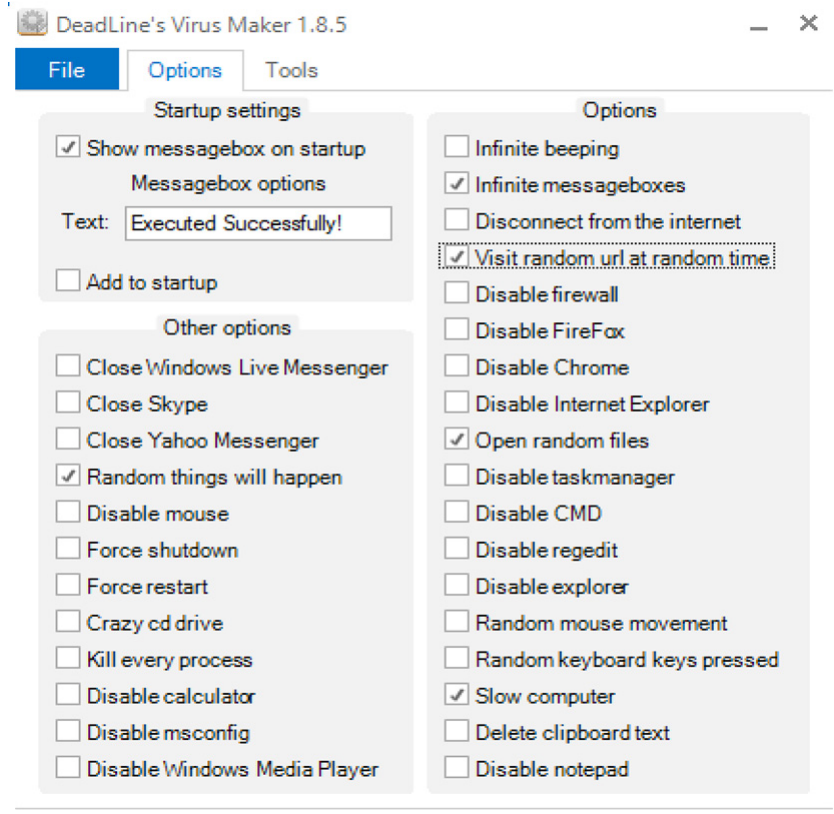

Figure 3. Screenshot of DeadLine Virus Maker tool. 
During the experiment, three different datasets were generated: one from the sensor of the cluster and one from the sensor of each desktop. The files were merged to one unified dataset that was used in this research. The dataset (see supplementary files) contains eleven fields. The first field indicates the date and time of the recording. The second and fifth represent the IDs of the running applications of desktop 1 and 2. Fields three and seven contain the current (I) measured (in amperes) of the two desktop computers every second respectively. Fields four and eight contains the value of the power consumption (in Watts) of the two of the desktops every second. The sixth field, which is of Boolean type, represents the presence (1) or absence (2) of a virus. The current and power consumption of the cluster of the two computers every second, is shown in fields nine and ten. The dataset as described here shows that there are various parameters, like current and power, obtained from the cluster. These indicators are obtained from various nodes of the cluster in order to precisely identified the application and the presence or absence of the virus.

Table 1. Application execution schedule.

\begin{tabular}{|c|c|c|c|c|c|}
\hline \multirow{2}{*}{$\#$} & \multirow{2}{*}{ Application Name/Action/Process } & \multicolumn{2}{|c|}{ 1st Day } & \multicolumn{2}{|c|}{ 2nd Day } \\
\hline & & Start Time & End Time & Start Time & End Time \\
\hline 1 & Start of Experiment & 05:01 & & 05:35 & \\
\hline 2 & Open the virus on desktop 2-Sensor 2 & - & - & 08:00 & \\
\hline 3 & NetBeans & 08:00 & 09:00 & 08:05 & 09:00 \\
\hline 4 & Idle & 09:00 & 09:10 & 09:00 & $9: 10$ \\
\hline 5 & MS Word & $9: 10$ & $10: 00$ & 9:10 & $10: 00$ \\
\hline 6 & Idle & $10: 00$ & 10:07 & $10: 00$ & 10:07 \\
\hline 7 & YouTube $240 p$ & 10:07 & $10: 20$ & 10:07 & $10: 20$ \\
\hline 8 & YouTube 360p & $10: 21$ & $10: 35$ & $10: 21$ & $10: 35$ \\
\hline 9 & YouTube 1080p & $10: 36$ & $10: 50$ & $10: 36$ & $10: 50$ \\
\hline 10 & YouTube $1440 p$ & $10: 51$ & $11: 15$ & $10: 51$ & $11: 15$ \\
\hline 11 & YouTube 4K & $11: 15$ & $11: 25$ & $11: 15$ & $11: 25$ \\
\hline 12 & Idle & $11: 25$ & $11: 30$ & $11: 25$ & $11: 30$ \\
\hline 13 & MS Excel & $11: 30$ & $12: 00$ & $11: 30$ & $12: 00$ \\
\hline 14 & Idle & $12: 00$ & $13: 35$ & $12: 00$ & $13: 35$ \\
\hline 15 & 3D Builder (Paint 3D) & $13: 35$ & $14: 20$ & $13: 35$ & $14: 20$ \\
\hline 16 & Idle & $14: 21$ & $14: 40$ & $14: 21$ & $14: 40$ \\
\hline 17 & $\begin{array}{c}\text { Copy Large Files 1st Half } \\
\text { From USB to PC }\end{array}$ & $14: 40$ & 15:05 & $14: 40$ & $15: 05$ \\
\hline 18 & Idle & $15: 05$ & $15: 40$ & $15: 05$ & $15: 40$ \\
\hline 19 & $\begin{array}{l}\text { Copy Large Files 1st Half } \\
\text { From PC to USB }\end{array}$ & $15: 40$ & $16: 00$ & $15: 40$ & $16: 00$ \\
\hline 20 & Idle & 16:01 & $16: 10$ & $16: 01$ & $16: 10$ \\
\hline 21 & $\begin{array}{c}\text { Game (The great unknown Houdini's } \\
\text { castle) (Game Crashed at Desktop } \\
\text { 2-Then Opened again with no issue) }\end{array}$ & $16: 11$ & $16: 50$ & $16: 11$ & $16: 50$ \\
\hline 22 & Idle & $16: 51$ & $17: 00$ & $16: 51$ & $17: 00$ \\
\hline 23 & Fusion 360 & $17: 00$ & $17: 41$ & $17: 00$ & $17: 41$ \\
\hline 24 & Idle & $17: 41$ & 18:00 & $17: 41$ & 18:00 \\
\hline 25 & MS PowerPoint & $18: 00$ & $18: 30$ & $18: 00$ & $18: 30$ \\
\hline 26 & Idle & $18: 31$ & $18: 35$ & $18: 31$ & $18: 35$ \\
\hline 27 & Web Browsing & $18: 36$ & 19:00 & $18: 36$ & 19:00 \\
\hline 28 & Idle & 19:00 & $05: 35$ & $19: 00$ & $05: 35$ \\
\hline 29 & End of Experiment & & $05: 35$ & & $05: 35$ \\
\hline
\end{tabular}

\subsection{Proposed Methodology}

The steps involved in the proposed methodology from preprocessing stage to the implications of various factors, along with time-series is described in this section. In order to achieve the objective 1 ("detect if a node and/or a cluster is infected"), descriptive analysis in the form of mean and standard deviation were used. To further emphasis our findings, variances of these measures were compared 
using an F-test. Objective 2 ("differentiate between different types of applications"), which is related to different applications, was analyzed using two-way ANOVA to understand whether there is an interaction between the virus and variety of applications. Furthermore, objective 3 ("detect occupancy of a node and/or a cluster), which is related to the occupancy of the node/cluster, can be predicted or identified using time-series based ARIMA method.

\subsubsection{Preprocessing}

The obtained dataset is obtained from sensors, where outlines or invalid data is possible. The preprocessing of the dataset was performed manually by removing certain outlines or mistakes introduced during the data collection process, such as nonarrival of data from a sensor, negative values, etc.

\subsubsection{Descriptive Analysis}

Various salient features such as mean and standard deviation were used to compare the factors involved in our experimentation. In order to validate the variance in the given factor, F-test and ANOVA are performed as described in Sections 2.2.3 and 2.2.4

\subsubsection{Comparing Power Consumption with and without Virus}

We studied the difference in variances between the power values when the virus is present or absent. Such an analysis is critical to recognize whether the interval (upper and lower bound) of power consumption for any specific application overlaps during the presence or absence of virus. Nonoverlapping of intervals with appropriate significance level will provide the necessary confidence to recognize the presence or absence of the virus. This was analyzed using a two samples of variances F-test with standard 95\% significance level.

\subsubsection{Implications of Three Factors}

Virus, application and power: having studied the differences in power consumption and the respective variances between different applications, with and without the running of the virus, we wished to see the implications of the factors involved in this study. Two-way ANOVA was used to analyze the dataset. As we have two factors (virus and application ID) involved, and the dependent variable is power. Data obtained from various sensors are analyzed further to validate the following hypotheses.

1. Mean values of the observations due to one factor (presence or absence of virus) are the same.

2. Mean values of the observations due to another factor (difference in applications: multimedia related, office related, idle) are the same.

3. There is no interaction between the two factors (presence/absence of virus and variety of applications).

\subsubsection{Time Series}

In order to perform ARIMA [31], the following three parameters need to be obtained:

- $\quad$ : The number of previous/lagged $Y$ values considered in our model for each time point. $p$ indicates autoregressive component;

- $\quad \mathrm{d}$ : The number of differences considered in our model to follow stationarity;

- $\quad \mathrm{q}$ : The number of previous/lagged error values considered in our model for each time point. 
Autocorrelation functions (both complete and partial), named as ACF and PACF, were used to obtain these parameters. Meanwhile, automatic forecasting algorithms could be used to identify the parameters for ARIMA. Akaike's information criterion (AIC) was used to select the appropriate model.

$$
A I C=L^{*}\left(\hat{\theta}, \hat{x}_{0}\right)+2 q
$$

where $q$ represents the number of parameters in $\theta$ plus the number of free states in $x_{0}, \hat{\theta}$ and $\hat{x}_{0}$ are the estimates of $\theta$ and $\hat{x}_{0}$.

The choice of AIC, shown in Equation (4), over other measures like MSE (mean square error) or MAPE (mean absolute percentage error) was justified because AIC considers the likelihood instead of one-step forecasts. Also, AIC was suitable for the selection among additive and multiplicative models.

Nonseasonal $\operatorname{ARIMA}(\mathrm{p}, \mathrm{d}, \mathrm{q})$ is given as:

$$
\phi(B)\left(1-B^{d}\right) y_{t}=c+\theta(B) \varepsilon_{t}
$$

where $\left\{\varepsilon_{t}\right\}$ is white noise, $B$ is backshift operator, $\phi(B)$ and $\theta(B)$ are polynomials of order $p$ and $q$ respectively.

Seasonal ARIMA $(p, d, q)(P, D, Q)_{m}$ is given as:

$$
\Phi\left(B^{m}\right) \phi(B)\left(1-B^{m}\right)^{D}(1-B)^{d} y_{t}=c+\Theta\left(B^{m}\right) \theta(B) \varepsilon_{t}
$$

where $m$ is the seasonal frequency, $\Phi\left(B^{m}\right)$ and $\Theta\left(B^{m}\right)$ are polynomials of order $P$ and $Q$ respectively.

$$
A I C=-2 \log (L)+2(p+q+P+Q+k)
$$

where $L$ is the maximum likelihood of the model fitted to the differenced data $\left(1-B^{m}\right)^{D}(1-B)^{d} y_{t}$.

\section{Results and Discussion}

\subsection{Preprocessing}

Before proceeding towards analyzing the obtained dataset, certain preliminary preprocessing revealed that, out of the 16 applications, along with an idle condition, all except one application was tested with both the presence and absence of virus. The application which represents opening the virus file could not be performed in the absence of the virus. The summary of the dataset collected is provided in Table 2. As an example, the number of records obtained from inactive virus scenario with an idle condition (application ID of zero) was 55630, while that of active virus scenario was 47929. In order to make the comparison effective, randomized selection of records for each application was performed. All the records were made equal to the record size of 494, which was the minimum record size available in the original dataset. The application number was 16 , which means opening of the virus file was removed during the preprocessing stage.

Table 2. Summary of dataset.

\begin{tabular}{cccccc}
\hline & Original Dataset & \multicolumn{3}{c}{ Preprocessed Dataset } \\
\hline Application ID & Inactive Virus & Active Virus & Application ID & Inactive Virus & Active Virus \\
\hline 0 & 55,630 & 47,929 & 0 & 494 & 494 \\
1 & 3123 & 3235 & 1 & 494 & 494 \\
2 & 2624 & 2869 & 2 & 494 & 494 \\
3 & 771 & 704 & 3 & 494 & 494 \\
4 & 736 & 889 & 4 & 494 & 494 \\
5 & 846 & 884 & 5 & 494 & 494 \\
\hline
\end{tabular}


Table 2. Cont.

\begin{tabular}{cccccc}
\hline & Original Dataset & \multicolumn{3}{c}{ Preprocessed Dataset } \\
\hline Application ID & Inactive Virus & Active Virus & Application ID & Inactive Virus & Active Virus \\
\hline 6 & 1216 & 1173 & 6 & 494 & 494 \\
7 & 597 & 494 & 7 & 494 & 494 \\
8 & 1626 & 1715 & 8 & 494 & 494 \\
9 & 2576 & 2674 & 9 & 494 & 494 \\
10 & 1160 & 1179 & 10 & 494 & 494 \\
11 & 1410 & 1416 & 11 & 494 & 494 \\
12 & 2338 & 2029 & 12 & 494 & 494 \\
13 & 2387 & 1934 & 13 & 494 & 494 \\
14 & 1524 & 1823 & 14 & 494 & 494 \\
15 & 1475 & 1282 & 15 & 494 & 494 \\
16 & 0 & 126 & & & \\
\hline
\end{tabular}

\subsection{Descriptive Analysis}

As shown in Table 3, descriptive analysis of the obtained data was performed to find the average and standard deviation of current and power values obtained from all the three sensors. In order to make sure that there was no other factor involved in this analysis, data analyzed here were taken only from a computer. The objective of this task was to compare the average and standard deviation between two computers running the same application(s) but with and without the virus running on them. Outcome analysis revealed that the average and standard deviation of current and power differed when the virus was running. For example, when the virus was active, application ID 2 consumed an average power of $61.78 \mathrm{~W}$ while that of the inactive virus consumed $43.69 \mathrm{~W}$. The range due to average +/- standard deviation are quite apart each other. This basically confirms that there was a clear difference in power consumption when virus was active or inactive in a system. Also, this could help in recognizing whether a computer is currently being used or not. In terms of power consumption, the applications consume higher to lower power in this order: multimedia and design applications, Microsoft Office applications and an idle condition.

Table 3. Descriptive analysis.

\begin{tabular}{ccccccccc}
\hline $\begin{array}{c}\text { Application } \\
\text { ID }\end{array}$ & $\begin{array}{c}\text { AVE (No } \\
\text { Virus): } \\
\text { Current }\end{array}$ & $\begin{array}{c}\text { STDEV } \\
\text { (No } \\
\text { Virus): } \\
\text { Current }\end{array}$ & $\begin{array}{c}\text { AVE } \\
\text { (Virus): } \\
\text { Current }\end{array}$ & $\begin{array}{c}\text { STDEV } \\
\text { (Virus): } \\
\text { Current }\end{array}$ & $\begin{array}{c}\text { AVE (No } \\
\text { Virus): } \\
\text { Power }\end{array}$ & $\begin{array}{c}\text { STDEV } \\
\text { (No } \\
\text { Virus): } \\
\text { Power }\end{array}$ & $\begin{array}{c}\text { AVE } \\
\text { (Virus): } \\
\text { Power }\end{array}$ & $\begin{array}{c}\text { STDEV } \\
\text { (Virus): } \\
\text { Power }\end{array}$ \\
\hline 0 & 0.274250333 & 0.028103 & 0.347148 & 0.020521 & 41.65125 & 7.485862 & 60.35578 & 4.995615 \\
1 & 0.30206212 & 0.040198 & 0.279725 & 0.032956 & 49.17003 & 10.83542 & 43.31901 & 8.913672 \\
2 & 0.282293826 & 0.029435 & 0.351423 & 0.019544 & 43.69131 & 7.785045 & 61.7839 & 5.003486 \\
3 & 0.294744488 & 0.027763 & 0.349382 & 0.016515 & 47.10895 & 7.176698 & 60.8821 & 4.130037 \\
4 & 0.338485054 & 0.282622 & 0.352319 & 0.018267 & 47.13043 & 9.747805 & 61.50731 & 4.612483 \\
5 & 0.310338061 & 0.027774 & 0.347696 & 0.017278 & 51.02364 & 7.14727 & 60.18891 & 4.235889 \\
6 & 0.328525493 & 0.02398 & 0.351409 & 0.018236 & 55.54605 & 6.243444 & 60.99829 & 4.507483 \\
7 & 0.336469012 & 0.045425 & 0.360682 & 0.014323 & 59.01173 & 12.08047 & 63.31984 & 3.612988 \\
8 & 0.276551661 & 0.028898 & 0.374414 & 0.015795 & 42.26999 & 7.718847 & 66.68105 & 3.88115 \\
9 & 0.285190606 & 0.031642 & 0.346214 & 0.017018 & 44.44293 & 8.223566 & 59.27786 & 4.086996 \\
10 & 0.304116379 & 0.030956 & 0.355818 & 0.020229 & 49.19138 & 7.936956 & 61.79389 & 4.938064 \\
11 & 0.299434043 & 0.029658 & 0.352472 & 0.019252 & 48.02128 & 7.655155 & 60.73729 & 4.650185 \\
12 & 0.342401625 & 0.025261 & 0.353526 & 0.020993 & 58.9337 & 6.272336 & 61.61656 & 5.078656 \\
13 & 0.289771261 & 0.030464 & 0.374752 & 0.023172 & 45.81232 & 8.066154 & 66.72285 & 5.671899 \\
14 & 0.282875328 & 0.029596 & 0.350807 & 0.018327 & 43.95276 & 8.015767 & 61.19748 & 4.584067 \\
15 & 0.300162712 & 0.035122 & 0.345573 & 0.017133 & 48.56271 & 9.288857 & 59.97738 & 4.294064 \\
\hline
\end{tabular}




\subsection{Comparing Power Consumption with and without Virus}

A sample of variance comparison for idle (no application) and application 15 is provided in Table 4. The comparison of F-critical with F (F ratio) value and the $p$-value with alpha, rejects the null hypothesis that their variances are the same. For example, in the case of idle condition, when no application was running, the F-critical value was 1.0123 and the F (F ratio) value was 2.6722. As the F-critical value is smaller than $\mathrm{F}$ (ratio) value, we conclude that the variances between the power with and without virus running is not the same. Also, $p$-value is smaller than the default alpha value of 0.05 . Similar results are obtained from other applications as well.

Table 4. F-Test of two samples of variance.

\begin{tabular}{cccccc}
\hline \multicolumn{2}{c}{ Variance of Power in Idle Condition } & \multicolumn{2}{c}{ Variance of Power with Application 3 } \\
\hline & Variable 1 & Variable 2 & & Variable 1 & Variable 2 \\
\hline Mean & 39.93072 & 60.02728 & Mean & 58.67635 & 60.01577 \\
Variance & 112.9857 & 42.28129 & Variance & 42.94666 & 18.63846 \\
Observations & 72,354 & 72,354 & Observations & 1205 & 1205 \\
df (Degrees of Freedom) & 72,353 & 72,353 & df (Degrees of freedom) & 1204 & 1204 \\
F (F ratio) & 2.672238 & & F (F ratio) & 2.304196 & \\
$\mathrm{P}(\mathrm{F}<$ = f) one-tail & 0 & & $\mathrm{P}(\mathrm{F}<\mathrm{f}$ f) one-tail & $1.53 \times 10^{-46}$ \\
$\mathrm{~F}-$ Critical one-tail & 1.012305 & & F-Critical one-tail & 1.099489 \\
\hline
\end{tabular}

\subsection{Implications of Three Factors}

Table 5 reveals the outcome of two-way ANOVA analysis. For the presence or absence of virus, when we compare F-Critical with F (F ratio), F-Critical (3.84) is smaller compared to F (F ratio) (2603.69), thus we reject the null hypothesis that the mean of the observations due to presence or absence of virus is not the same. Also, the obtained $p$-value is much smaller than that of alpha (0.05). This confirms our idea that the power consumption is affected due to running of virus program in a computer.

With regards to the variety among the applications, the F-critical (3.84) is smaller than the F (F ratio) value (302277.3). Thus, we reject the null hypothesis that the mean values of the applications are the same. It is clear that, from our study, the application is running at any point of time could easily be identified.

Similarly, the third hypothesis, which indicates that there is no interaction between virus and applications, is also rejected. Here, there is a clear indication that the impact of virus depends on the respective application. Thus, the rate of change in power consumption varies from one application to other, when the virus is executed.

In summary, the following is determined:

1. Viruses have an impact on the power;

2. A variety of applications impact the power consumption of the system;

3. There is an interaction between the virus and the application with regards to the impact on the system.

Table 5. Two-way ANOVA.

\begin{tabular}{ccccccc}
\hline \multicolumn{7}{c}{ ANOVA } \\
Source of \\
Variation & $\begin{array}{c}\text { SS (Sum of } \\
\text { Squares) }\end{array}$ & $\begin{array}{c}\text { df (Degrees } \\
\text { of Freedom) }\end{array}$ & $\begin{array}{c}\text { MS (Mean } \\
\text { Squares) }\end{array}$ & F (F Ratio) & $\boldsymbol{p}$-Value & F-Critical \\
\hline Virus & $150,880.9$ & 1 & $150,880.9$ & 2603.692 & 0 & 3.841753 \\
Application & $17,516,617$ & 1 & $17,516,617$ & 302277.3 & 0 & 3.841753 \\
Interaction & $104,200.9$ & 1 & $104,200.9$ & 1798.154 & 0 & 3.841753 \\
Within & $1,831,878$ & 31,612 & 57.94883 & & & \\
Total & $19,603,577$ & 31,615 & & & & \\
\hline
\end{tabular}


A similar two-way ANOVA was performed after grouping the applications into nine groups like idle, programming, multimedia, design, general, etc. The outcome of such an analysis is provided in Table 6. Similar to the outcome in Table 5, Table 6 also rejects the null hypothesis. This confirms the impact of virus on power along with the respective group of application being executed.

Table 6. Two-way ANOVA.

\begin{tabular}{|c|c|c|c|c|c|c|}
\hline \multicolumn{7}{|c|}{ ANOVA } \\
\hline $\begin{array}{l}\text { Source of } \\
\text { Variation }\end{array}$ & $\begin{array}{c}\text { SS (Sum of } \\
\text { Squares) }\end{array}$ & $\begin{array}{l}\text { df (Degrees } \\
\text { of Freedom) }\end{array}$ & $\begin{array}{c}\text { MS (Mean } \\
\text { Squares) }\end{array}$ & F (F Ratio) & $p$-Value & F-Critical \\
\hline Virus & 2429.457 & 1 & 2429.457 & 39.57663 & $3.21 \times 10^{-10}$ & 3.841853 \\
\hline Application & $17,168,455$ & 1 & $17,168,455$ & 279679.6 & 0 & 3.841853 \\
\hline Interaction & 2429.457 & 1 & 2429.457 & 39.57663 & $3.21 \times 10^{-10}$ & 3.841853 \\
\hline Within & $1,448,467$ & 23,596 & 61.38615 & & & \\
\hline Total & $18,621,781$ & 23,599 & & & & \\
\hline
\end{tabular}

\subsection{Time Series}

Having collected the data for two days while applying various activities with and without the virus, a time-series-based analysis was also performed. Time series is composed of trend, seasonal and cyclic components. Before performing ARIMA (autoregressive integrated moving average) on the time series, the assumption of stationarity was evaluated. An augmented Dickey-Fuller test was performed to test for stationarity. The results reveal the dataset, power consumption data specifically, follows stationarity, with a $p$-value of 0.01 , which is less than the alpha value of 0.05 . Smooth moving average was performed over the given power data. Averaging over a time period of $20 \mathrm{~min}$ returns the trend of the curve as in Figure 4.

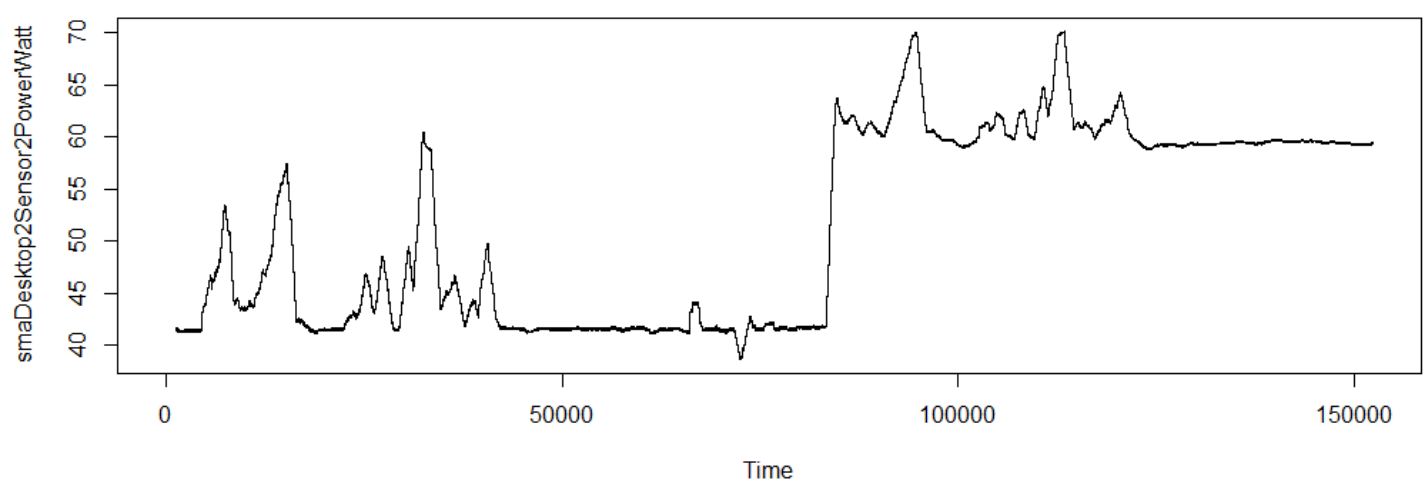

Figure 4. Smooth moving average with $n=1200$ (20 min).

The upward trend at a certain time indicates the impact of running a virus on the power consumption. Decomposition of the time-series into trend, seasonal, and random components reveals that there is generally a trend component in the obtained time-series data. Excluding trend, the seasonality is not prevalent in the dataset. Also, the random component does not seem to highly impact on the data. Figure 5 shows the decomposition of additive time series. 


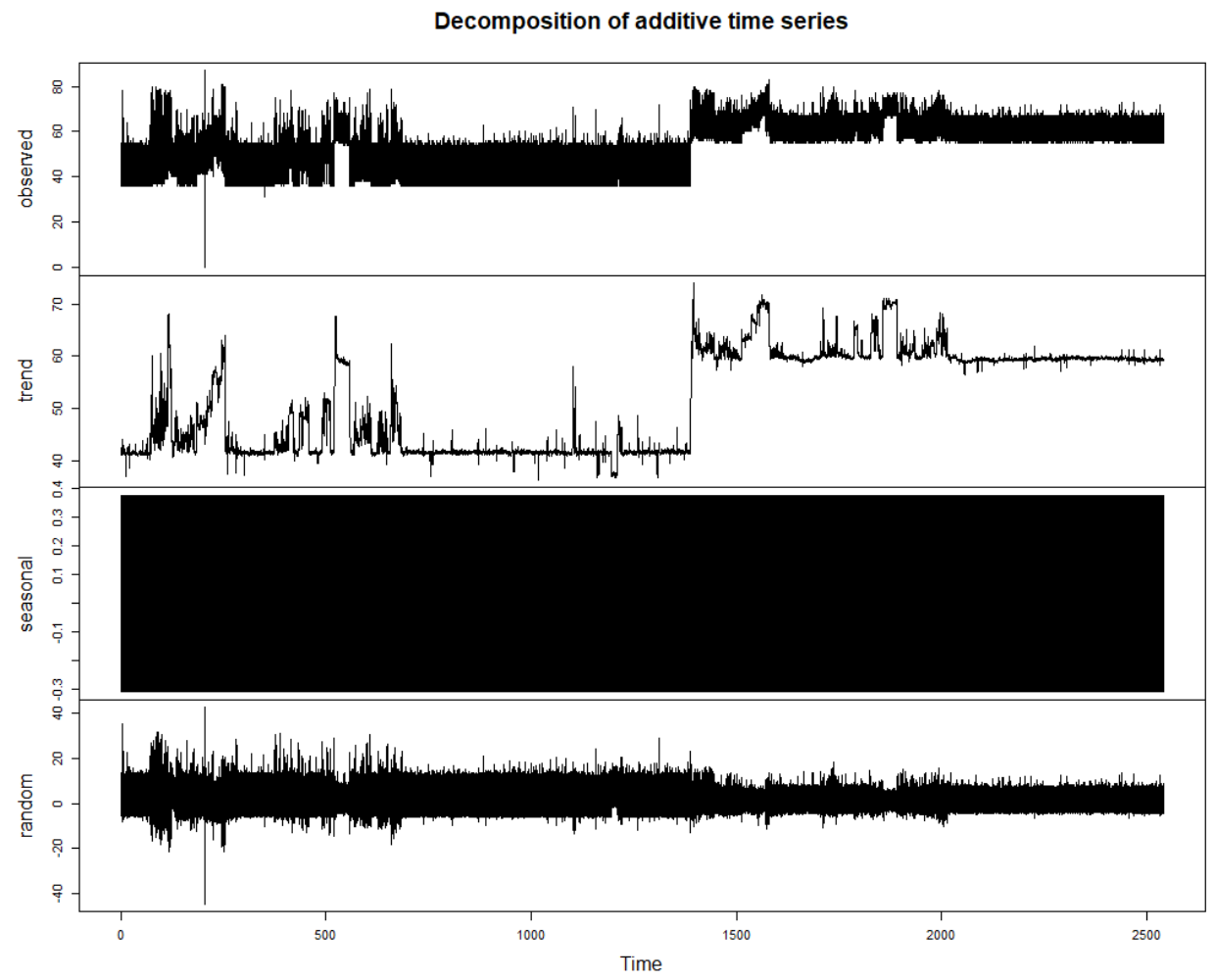

Figure 5. Decomposition of the time series dataset.

Based on our experimental analysis, ARIMA $(2,0,0)(1,1,0)[60]$ with drift was found to be the most suitable model. This model obtained an AIC of 792012.8. The value of $p$ was 2 , which indicates the relationship between an observation with that of the one preceding the one immediately preceding it. The zero value of $d$ indicates that the integrative component was absent. The value of zero for $q$ indicates no relationship between an error and the ones preceding it. The presence of seasonality in the model indicates repetitions of the same events. In our analysis, the repetitions or seasonality depends on the time at which certain applications or the virus were executed. As in real time such an event varies from one situation to another, the seasonality value indicated by the proposed model was not significant in this study.

Forecasting for a time period of $3000 \mathrm{~s}$ was done using the selected model as shown in Figure 6. Plot shows that the pattern is expected to remain in the same level with the upper and lower bound tending to vary.

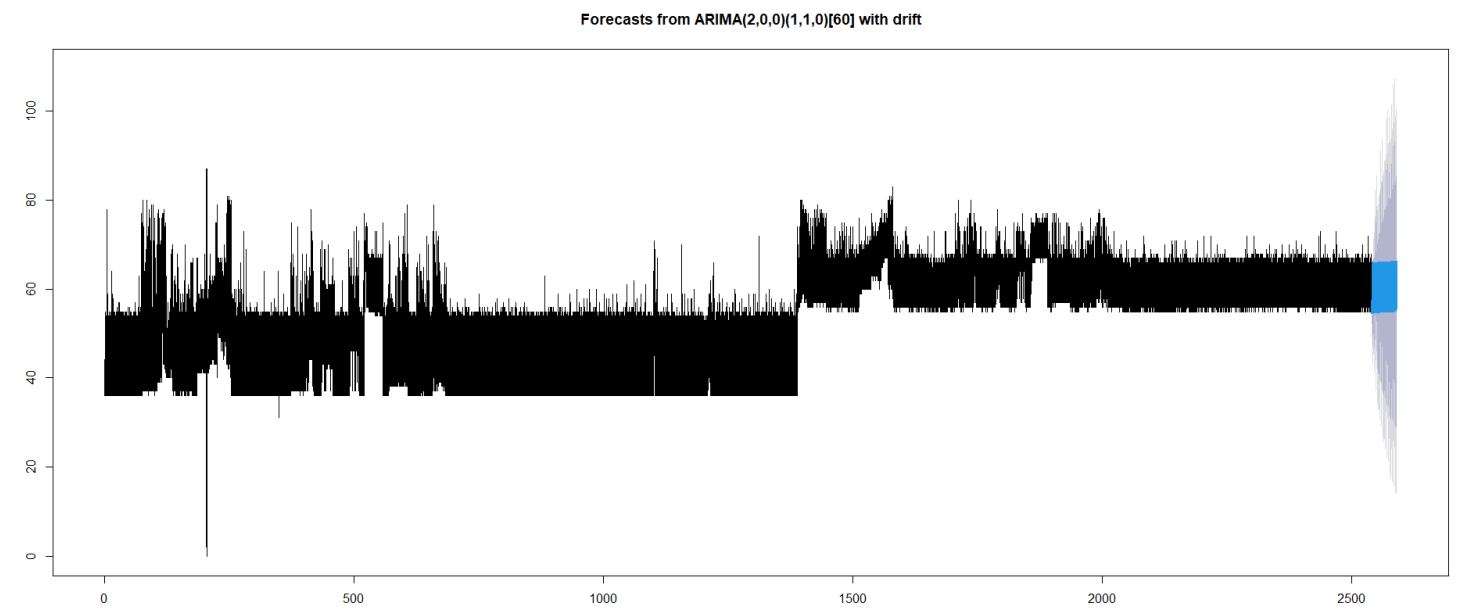

Figure 6. Forecast using ARIMA. 


\subsection{Outcomes from the Analysis}

Overall, this study has provided the following insights:

1. Descriptive analysis: Average and standard deviation of power consumption varied while running any specific application, with or without virus;

2. F-Test of two samples of variances: variances of power consumption varied with the presence or absence of virus;

3. Two-way ANOVA: The interaction between the presence or absence of virus and the specific application running does impact the power consumption on the computer. Power consumption of the computer also varied due to the type of the application and whether the virus was running or not;

4. Time-Series: Time series analysis on the dataset reveals that the power consumption can be represented with ARIMA model using autoregression.

\section{Conclusions}

This paper presented a methodology for detecting potentially infected computers and cluster of computers connected on a smart grid by analyzing their power consumption data. The research used data of a cluster of two computers connected to power measurements sensors. The data were analyzed using descriptive analysis, two-way ANOVA and ARIMA. The results showed that the presence/absence of virus and its interaction with variety of applications does affect the power consumption. We were able to detect infected nodes and clusters. Additionally, we can detect what type of application is running on the machine. It is possible to accurately detect physical or remote occupancy of the lab as there is a significant variation of the power usage. All these points make this work useful for facility management, lab administrations and many more. This research can be generalized to any context with the proposed experiment architecture capable of facilitating individualistic data acquisition, hence allowing the system to produce diagnostics. The main limitation of this work lies with the number of executed applications during the experiment and the limited number of nodes and duration. However, the aim of this research was to test the feasibility of such an approach, something that was proven based on the above results. Future work will mainly focus on enhancing the dataset to overcome the above limitations. Additionally, we will detect normal and abnormal behaviors, for example, massive sudden power consumption from a PC that is currently used is a suspicious behavior.

Supplementary Materials: The dataset is available online at http://www.mdpi.com/1424-8220/20/18/5075/s1.

Author Contributions: Conceptualization, G.T. and M.A.; methodology, G.T., A.O.K. (Adil Omar Khadidos) and S.M.B.; software, M.A.; validation, F.Q.K. and A.O.K. (Alaa Omar Khadidos); investigation, M.A., G.T., F.Q.K., A.O.K. (Alaa Omar Khadidos), A.O.K. (Adil Omar Khadidos) and S.M.B.; resources, A.O.K. (Adil Omar Khadidos) and A.O.K. (Alaa Omar Khadidos); data curation, M.A. and A.O.K. (Adil Omar Khadidos); writing-original draft preparation, M.A., G.T., S.M.B. and A.O.K. (Adil Omar Khadidos); writing-review and editing, S.M.B., M.A., F.Q.K., G.T., A.O.K. (Adil Omar Khadidos); visualization, M.A. and S.M.B.; supervision, G.T. and A.O.K. (Adil Omar Khadidos); All authors have read and agreed to the published version of the manuscript.

Funding: This research received no external funding.

Conflicts of Interest: The authors declare no conflict of interest.

\section{References}

1. Alamaniotis, M.; Bourbakis, N.; Tsoukalas, L.H. Enhancing privacy of electricity consumption in smart cities through morphing of anticipated demand pattern utilizing self-elasticity and genetic algorithms. Sustain. Cities Soc. 2019, 46, 101426. [CrossRef]

2. Talaat, M.; Alsayyari, A.S.; Alblawi, A.; Hatata, A.Y. Hybrid-cloud-based data processing for power system monitoring in smart grids. Sustain. Cities Soc. 2020, 55, 102049. [CrossRef]

3. Marinakis, V.; Karakosta, C.; Doukas, H.; Androulaki, S.; Psarras, J. A building automation and control tool for remote and real time monitoring of energy consumption. Sustain. Cities Soc. 2013, 6, 11-15. [CrossRef] 
4. Roselyn, J.P.; Uthra, R.A.; Raj, A.; Devaraj, D.; Bharadwaj, P.; Krishna Kaki, S.V.D. Development and implementation of novel sensor fusion algorithm for occupancy detection and automation in energy efficient buildings. Sustain. Cities Soc. 2019, 44, 85-98. [CrossRef]

5. Luckett, P.; McDonald, J.T.; Glisson, W.B.; Benton, R.; Dawson, J.; Doyle, B.A. Identifying stealth malware using CPU power consumption and learning algorithms. J. Comput. Secur. 2018, 26, 589-613. [CrossRef]

6. Hernandez Jimenez, J.; Goseva-Popstojanova, K. Malware Detection Using Power Consumption and Network Traffic Data. In Proceedings of the 2019 2nd International Conference on Data Intelligence and Security (ICDIS), South Padre Island, TX, USA, 28-30 June 2019; pp. 53-59.

7. Ioaneş, A.; Tirnovan, R. Power Grid Health Assessment Using Machine Learning Algorithms. In Proceedings of the 2019 11th International Symposium on Advanced Topics in Electrical Engineering (ATEE), Bucharest, Romania, 28-30 March 2019; pp. 1-4.

8. Mehrotra, D.; Srivastava, R.; Nagpal, R.; Nagpal, D. Multiclass classification of mobile applications as per energy consumption. J. King Saud Univ. Comput. Inf. Sci. 2018. [CrossRef]

9. Android Anomaly Detection System uSing Machine Learning Classification-IEEE Conference Publication. Available online: https://ieeexplore.ieee.org/document/7352512 (accessed on 24 August 2020).

10. Zefferer, T.; Teufl, P.; Derler, D.; Potzmader, K.; Oprisnik, A.; Gasparitz, H.; Höller, A. Power Consumption-based Application Classification and Malware Detection on Android Using Machine-Learning Techniques. In Proceedings of the Future Computing 2013: The Fifth International Conference on Future Computational Technologies and Applications, IARIA, Valencia, Spain, 27 May-1 June 2013; pp. $26-31$.

11. Electrical Devices Identification through Power Consumption Using Machine Learning Techniques | Semantic Scholar. Available online: https://www.semanticscholar.org/paper/Electrical-Devices-Identificationthrough-Power-Abeykoon-Kankanamdurage/f0ea3eabe628010a092f505b64ce26f48e82424f (accessed on 24 August 2020).

12. Moon, J.; Park, J.; Hwang, E.; Jun, S. Forecasting power consumption for higher educational institutions based on machine learning. J. Supercomput. 2018, 74, 3778-3800. [CrossRef]

13. Shao, M.; Wang, X.; Bu, Z.; Chen, X.; Wang, Y. Prediction of energy consumption in hotel buildings via support vector machines. Sustain. Cities Soc. 2020, 57, 102128. [CrossRef]

14. Gajowniczek, K.; Zabkowski, T. Two-Stage Electricity Demand Modeling Using Machine Learning Algorithms. Energies 2017, 10, 1547. [CrossRef]

15. Markovič, R.; Gosak, M.; Grubelnik, V.; Marhl, M.; Virtič, P. Data-driven classification of residential energy consumption patterns by means of functional connectivity networks. Appl. Energy 2019, 242, 506-515. [CrossRef]

16. Iqbal, J.; Khan, M.; Talha, M.; Farman, H.; Jan, B.; Muhammad, A.; Khattak, H.A. A generic internet of things architecture for controlling electrical energy consumption in smart homes. Sustain. Cities Soc. 2018, 43, 443-450. [CrossRef]

17. Bourdeau, M.; Zhai, X.; Nefzaoui, E.; Guo, X.; Chatellier, P. Modeling and forecasting building energy consumption: A review of data-driven techniques. Sustain. Cities Soc. 2019, 48, 101533. [CrossRef]

18. Croce, D.; Giuliano, F.; Tinnirello, I.; Giarré, L. Privacy-Preserving Overgrid: Secure Data Collection for the Smart Grid. Sensors 2020, 20, 2249. [CrossRef]

19. Zhang, G.; Ji, X.; Li, Y.; Xu, W. Power-Based Non-Intrusive Condition Monitoring for Terminal Device in Smart Grid. Sensors 2020, 20, 3635. [CrossRef]

20. Lee, Y.; Lee, Y.S. A Low-Cost Surge Current Detection Sensor with Predictive Lifetime Display Function for Maintenance of Surge Protective Devices. Sensors 2020, 20, 2310. [CrossRef]

21. Blazakis, K.V.; Kapetanakis, T.N.; Stavrakakis, G.S. Effective Electricity Theft Detection in Power Distribution Grids Using an Adaptive Neuro Fuzzy Inference System. Energies 2020, 13, 3110. [CrossRef]

22. Cibinskiene, A.; Dumciuviene, D.; Andrijauskiene, M. Energy Consumption in Public Buildings: The Determinants of Occupants' Behavior. Energies 2020, 13, 3586. [CrossRef]

23. Oh, J. IoT-Based Smart Plug for Residential Energy Conservation: An Empirical Study Based on 15 Months' Monitoring. Energies 2020, 13, 4035. [CrossRef]

24. Myridakis, D.; Spathoulas, G.; Kakarountas, A.; Schinianakis, D. Smart Devices Security Enhancement via Power Supply Monitoring. Future Internet 2020, 12, 48. [CrossRef] 
25. Moradzadeh, A.; Sadeghian, O.; Pourhossein, K.; Mohammadi-Ivatloo, B.; Anvari-Moghaddam, A. Improving Residential Load Disaggregation for Sustainable Development of Energy via Principal Component Analysis. Sustainability 2020, 12, 3158. [CrossRef]

26. Mahia, F.; Dey, A.R.; Masud, M.A.; Mahmud, M.S. Forecasting Electricity Consumption using ARIMA Model. In Proceedings of the 2019 International Conference on Sustainable Technologies for Industry 4.0 (STI), Dhaka, Bangladesh, 24-25 December 2019; pp. 1-6.

27. Ma, M.; Wang, Z. Prediction of the Energy Consumption Variation Trend in South Africa based on ARIMA, NGM and NGM-ARIMA Models. Energies 2020, 13, 10. [CrossRef]

28. Pérez, X. Configuration of Sonoff Sensor to Espurna Firmware. Available online: https://github.com/ xoseperez/espurna (accessed on 24 August 2020).

29. Running on Raspberry Pi: Node-RED. Available online: https://nodered.org/docs/getting-started/raspberrypi (accessed on 24 August 2020).

30. DeadLine's Virus Maker 1.8.5. Available online: http://superhackings.blogspot.com/2015/08/deadlines-virusmaker-185free-download.html (accessed on 24 August 2020).

31. Hyndman, R.J.; Khandakar, Y. Automatic Time Series Forecasting: The forecast Package for R. J. Stat. Softw. 2008, 27, 1-22. [CrossRef]

(C) 2020 by the authors. Licensee MDPI, Basel, Switzerland. This article is an open access article distributed under the terms and conditions of the Creative Commons Attribution (CC BY) license (http://creativecommons.org/licenses/by/4.0/). 